\title{
Stellar and ionized gas kinematics of the interacting Seyfert 1.9 galaxy NGC 2992
}

\author{
B. García-Lorenzo ${ }^{1}$, S. Arribas ${ }^{2,3}$, and E. Mediavilla ${ }^{4}$ \\ 1 Isaac Newton Group of Telescopes, Apdo de Correos 321, 38700 S/C de La Palma, Canary Islands, Spain \\ 2 Space Telescope Science Institute, 3700 San Martin Drive, Baltimore, MD 21218, USA Affiliated with the \\ Astrophysics Division, Space Science Department of ESA \\ e-mail: arribas@stsci.edu \\ 3 On leave from the Instituto de Astrofísica de Canarias-Consejo Superior de Investigaciones Científicas, Spain \\ 4 Instituto de Astrofísica de Canarias, 38205 La Laguna, Tenerife, Canary Islands, Spain \\ e-mail: emg@ll.iac.es
}

Received 13 March 2001 / Accepted 30 August 2001

\begin{abstract}
Integral field spectroscopy in the central $16^{\prime \prime} \times 12^{\prime \prime}\left(2.4 \mathrm{kpc} \times 1.8 \mathrm{kpc}\right.$, if $\left.H_{0}=75 \mathrm{~km} \mathrm{~s}^{-1} \mathrm{Mpc}^{-1}\right)$ of the Seyfert 1.9 galaxy NGC 2992 has been obtained using the fibre system INTEGRAL. The data are mainly used to study the stellar and ionized gas kinematics. In spite of the photometric disruptions in the outer parts $(r>$ $6 \mathrm{kpc}$ ) produced by the interaction with its close companion (NGC 2993), the present stellar velocity field shows regular rotation. The ionized gas presents several kinematically distinct components. Apart from the outflowing component already reported by other authors, we found an additional (high ionization) kinematic component which seems to be associated with the boundaries of the figure-of-eight-shaped emission detected in the $6 \mathrm{~cm}$ radio map. We locate the hidden nucleus in the apex of the biconical structure defined by the [O III] emission, coincident with the outflow origin and with the center of the dust lane. We do not find any clear evidence of direct influence of the interaction in the kinematics of the stars or the ionized gas in the circumnuclear region of NGC 2992.
\end{abstract}

Key words. instrumentation: spectrographs - galaxies: active - galaxies: individual: NGC 2992 galaxies: interactions - galaxies: kinematics and dynamics - galaxies: Seyfert

\section{Introduction}

NGC 2992 is a highly inclined $\left(i=70^{\circ}\right)$ southern nearby $\left(d \sim 31 \mathrm{Mpc}\right.$, if $\left.H_{0}=75 \mathrm{~km} \mathrm{~s}^{-1} \mathrm{Mpc}^{-1}\right)$ Sa galaxy, which can be sampled in reasonably fine detail $\left(1^{\prime \prime} \sim 150 \mathrm{pc}\right)$. With NGC 2993, located $\sim 3^{\prime} \mathrm{SE}$, it forms an interacting system (Arp 245) connected by a tidal tail. NGC 2992 also shows another prominent tail to the north which is probably a consequence of the interaction. A dust lane cross the galaxy nearly parallel to the photometric major axis $\left(\sim 15^{\circ}, \mathrm{CR} 3\right)$ and dividing the bulge into two sections (see Fig. 1).

NGC 2992 was initially classified as Seyfert 2 (Osmer et al. 1974) on the basis of its high $[\mathrm{O}$ III] $/ \mathrm{H} \beta$ ratio, but later Ward et al. (1980) proposed that it is better classified as an intermediate Seyfert 1 galaxy considering the presence of a broad $\mathrm{H} \alpha$ and its identification with a

Send offprint requests to: B. García-Lorenzo, e-mail: bgarcia@ing.iac.es strong HEAO-1 X-ray source. More recently, Glass (1997) suggested that NGC 2992 is a hybrid between an intermediate Seyfert and a starburst galaxy, the latter being induced by the interaction with NGC 2993.

NGC 2992 represents an interesting case of a Seyfert galaxy in interaction and therefore gives us the opportunity to analyse how interaction affects the region around the AGN. Photometric studies are limited by the presence of the dust lane which enhances the relevance of multiwavelength kinematic studies for this galaxy. Previous kinematic studies of NGC 2992 have focused on the ionized gas in the circumnuclear region. They started with the work by Heckman et al. (1981), who reported quite complex kinematics on the basis of long-slit observations along $\mathrm{PA}=120^{\circ}$. This study was refined by Colina et al. (1987) (hereafter, CFKP87), who obtained long-slit spectroscopy along three position angles $\left(26^{\circ}, 130^{\circ}\right.$ and $\left.160^{\circ}\right)$. These authors distinguish between i) the nuclear region $\left(r<3^{\prime \prime}\right)$ where blue asymmetric profiles and line shifts with respect 
to the systemic were found, and ii) the off-nuclear region $\left(3^{\prime \prime}<r<20^{\prime \prime}\right)$ characterized by a complex pattern probably due to the dust lane and the effects of the companion. Further effort in this direction is due to Márquez et al. (1998) (hereafter, MBDP98), who report the presence of double-peaked line profiles confirming the complexity of the velocity field and propose a simple model with two kinematically distinct gaseous components (circular rotation + constant radial outflow). Recently, a detailed 2D kinematic study based on Fabry-Perot observations has been published (Veilleux et al. 2001 - hereafter, VSM01). NGC 2992 is also included in the sample of Seyfert galaxies with companions studied by Keel (1996) and in the edge-on Seyfert galaxies by Colbert et al. (1996).

The present paper focuses on the stellar and ionized gas kinematics in the circumnuclear region of NGC 2992 on the basis of two-dimensional (integral field) spectroscopy. This technique is particularly suited for such a study considering the complexity of NGC 2992. In particular, we will present simultaneous spectroscopy in 189 regions regularly distributed over $16^{\prime \prime} \times 12^{\prime \prime}(2.4 \mathrm{kpc} \times$ $1.8 \mathrm{kpc})$. The spectral range includes the $\mathrm{H} \beta-\left[\begin{array}{ll}\mathrm{O} & \mathrm{III}\end{array}\right]$ $\lambda \lambda 4959,5007$, and $\mathrm{H} \alpha+[\mathrm{N}$ II $] \lambda \lambda 6548,6584-[\mathrm{S}$ II $]$ $\lambda \lambda 6716,6731$ emission lines and the $\mathrm{Mg} \mathrm{I} b$, and $\mathrm{Na} \mathrm{I}$ $\lambda \lambda 5890,5896$ absorption lines at a resolution of $\sim 3 \AA$. This paper is part of a programme aimed at the study of the nuclear environment of active galaxies on the basis of integral field spectroscopy.

\section{Instrumentation, observations and data reduction}

\subsection{Observations and instrumentation}

The data analysed in this paper were obtained on 1998 April 4 and 5 at the Observatorio del Roque de los Muchachos (ORM) on the island of La Palma. Weather conditions during the first night were fairly good, with a seeing of about $1^{\prime \prime}$. However, the second night was cloudy with variable deep cirrus during the NGC 2992 observations. Both nights were bright with a crescent moon.

The $4.2 \mathrm{~m}$ William Herschel Telecope (WHT) was used in combination with the INTEGRAL fibre system (Arribas et al. 1998) and the WYFFOS spectrograph (Bingham et al. 1994).

INTEGRAL links the Nasmyth focus of the WHT with the slit of WYFFOS via three optical fibre bundles. These three bundles have different spatial configurations on the focal plane and can be interchanged online depending on the scientific programme or the prevailing seeing conditions. They can also be easily and quickly put into the telescope beam. At the focal plane the fibres of each bundle are arranged in two groups, one forming a rectangle, and the other an outer ring for collecting background light. The data discussed in this paper were obtained with the standard bundle 2, which consists of 219 fibres, each 0!'9 in diameter on the sky. The central rectangle is formed by 189 fibres covering an area of $16^{\prime \prime} \times 12^{\prime \prime} .3$ on the sky, and the other 30 fibres form a ring $90^{\prime \prime}$ in diameter. Figure 1 shows the actual distribution of the fibres in the focal plane.

The WYFFOS spectrograph was equipped with a 1200 groove $\mathrm{mm}^{-1}$ grating centred on $5000 \AA$ (first night) and $6305 \AA$ (second night). A Tek6 CCD array of $1124 \times$ 1124 pixels of $24 \mu \mathrm{m}$ size was used, giving a linear dispersion of about $1.45 \AA \mathrm{pixel}^{-1}$. With this configuration, and pointing to the central region of NGC 2992, we took five (six) exposures of $1800 \mathrm{~s}$ each during the first (second) night.

\subsection{Data reduction}

The reduction consists of two main steps: i) basic reduction of the spectra (i.e. bias, flatfielding, extraction, wavelength calibration, etc.) and ii) generation of maps of spectral features (e.g. line intensity, velocity fields, etc.) from the spectra.

Step i) was performed in the IRAF environment and following the standard procedures. We obtained typical wavelength calibration errors of $0.1 \AA$, which give velocity uncertainties of \pm 6 , and $\pm 4.5 \mathrm{~km} \mathrm{~s}^{-1}$ for [O III] $\lambda 5007$ and $\mathrm{H} \alpha$, respectively. We corrected the spectra for differential atmospheric refraction effects following the method proposed by Arribas et al. (1999). To apply this method we used the atmospheric temperature and pressure given by the Carlsberg Meridian Circle Catalogue (1998) and we estimate the differential atmospheric refraction according to the model given by Allen (1973).

For step ii) we used a Renka \& Cline two-dimensional interpolation method (E01SAF and E01SBF routines of the NAG Fortran Library Routine Document). In particular we transformed an ASCII file with the actual position of the fibres (accurately determined by a metrology machine), and the spectral feature corresponding to each fibre into a regularly spaced rectangular grid. In this way we built up images of $95 \times 95$ pixels with a scale $\sim 0.2^{\prime \prime}$ pixel $^{-1}$, which can be treated with standard astronomical software. Maps generated in this way are presented in Sect. 4 and beyond.

\section{The data}

Figure 2 shows the nuclear spectrum of NGC 2992 in the full wavelength range observed. This spectrum was obtained by averaging the seven spectra closer to the continuum peak $\left(r<1^{\prime \prime} \cdot 2\right)$, and it confirms the classification of NGC 2992 as an intermediate Seyfert galaxy. It is interesting to note how the faint broad $\mathrm{H} \alpha$ component has no counterpart in the $\mathrm{H} \beta$ line. This may be due to the presence of $\mathrm{H} \beta$ in absorption or, more probably, to the effects of extinction, which are more severe for $\mathrm{H} \beta$. The nuclear spectrum also shows prominent stellar absorption lines (e.g. Na I $\lambda \lambda 5890,5896)$. Figures $2 \mathrm{~b}-\mathrm{g}$ show the individual spectra corresponding to the 189 observed positions (fibres) in selected spectral ranges which include the $\mathrm{H} \beta$, [O III] $\lambda \lambda 4959,5007, \mathrm{Mg} \mathrm{I} b \lambda \lambda \lambda 5184,5173,5167$, $\mathrm{Na}$ I $\lambda \lambda 5890,5896, \mathrm{H} \alpha+[\mathrm{N}$ II $] \lambda \lambda 6548,6584$ and 

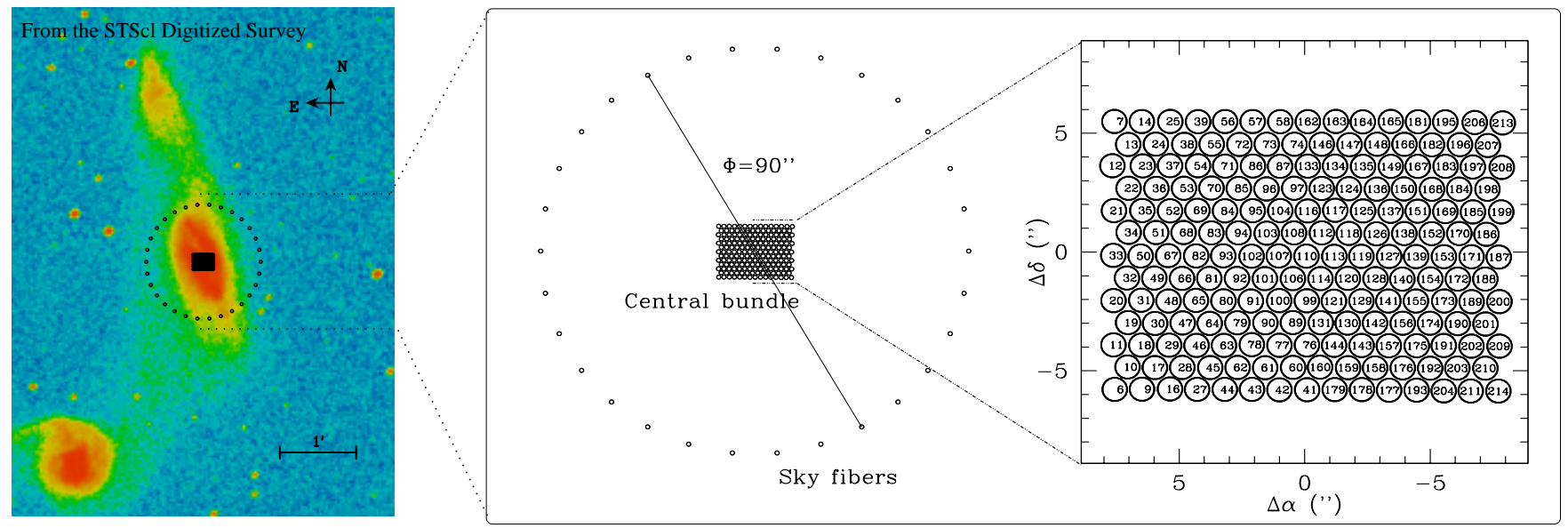

Fig. 1. Fibre distribution on the focal plane of the William Herschel Telescope. Numbers indicate the actual position of the fibres in the slit.
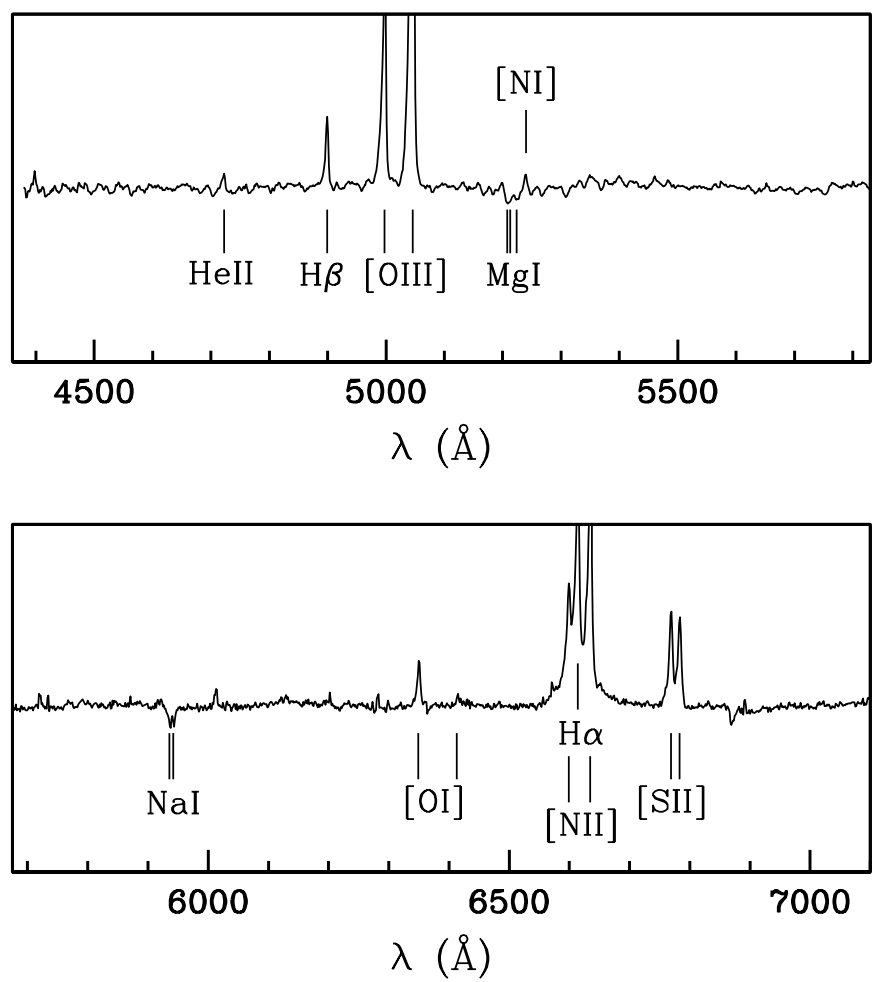

Fig. 2. a) Nuclear spectrum of NGC 2992 in the full spectral range observed (4360-5800 and 5675-7100 $)$.

[S II] $\lambda \lambda 6716,6731$ lines, respectively. In these figures the spectra at each location are autoscaled to better show the profile shape. Note how the profiles change from region to region.

The location of the dust lane crossing NGC 2992 is clearly traced by the spectra with lower $S / N$ (for instance, see in Fig. 2c spectra between those labelled 214 and 58). An inspection of the Figs. 2b,c,f and $g$ reveals asymmetric profiles and evidence of weak double peaks in different regions (see, for instance, the fibres labelled 106, 108 and 69 in Fig. 2c). Previous works have reported blue asymmetries and double-peaked profiles in the central region of the galaxy (MBDP98; CFKP87). The present data (which include no previously observed absorption or emission lines) confirm their findings and represent a unique and comprehensive set of data for analysing both the stellar and the ionized gas components in the inner region of NGC 2992. These spectra are publicly available at the following WWW address: http: /www . ing. iac.es/ bgarcia/NGC2992

\section{Stellar component}

\subsection{Stellar distribution: Continuum maps}

Figure 3 shows three continua directly obtained from the observed spectra after integrating and interpolating the signal in the spectral range indicated (see Sect. 2.2). Continuum peaks are in positional agreement within \pm 0 ". 1 and hereafter we will refer to this position as the optical nucleus (which we take as the origin of coordinates in all the figures). Figure 3 also includes a direct filter image (F606W) from the HST (WFPC2), which has been convolved with a two-dimensional Gaussian $\left(\sigma \sim 1^{\prime \prime}\right)$ in order to simulate the seeing during our observations. This map agrees remarkably well with our reddest continuum in spite of their different wavelengths.

NGC 2992 continua clearly show the dust lane splitting the bulge into two asymmetric sections with the optical nucleus located towards the E. The dust lane and isophotes in the observed region are aligned in the same direction. Fitting elliptical isophotes, we obtained a mean position angle of $34^{\circ} \pm 3^{\circ}$ for the photometric axis in the inner $5^{\prime \prime}$. This is in good agreement with determinations both from near-IR imaging (Alonso-Herrero et al. 1998) and kinematics (VSM01). However, this direction is far from that corresponding to the large-scale (outer) photometric axis $\left(\sim 15^{\circ}, \mathrm{RC} 3\right)$.

Although the elliptical fitting gives an inclination of about $66^{\circ}$, we are aware that the presence of the dust lane can strongly affect this estimate. 


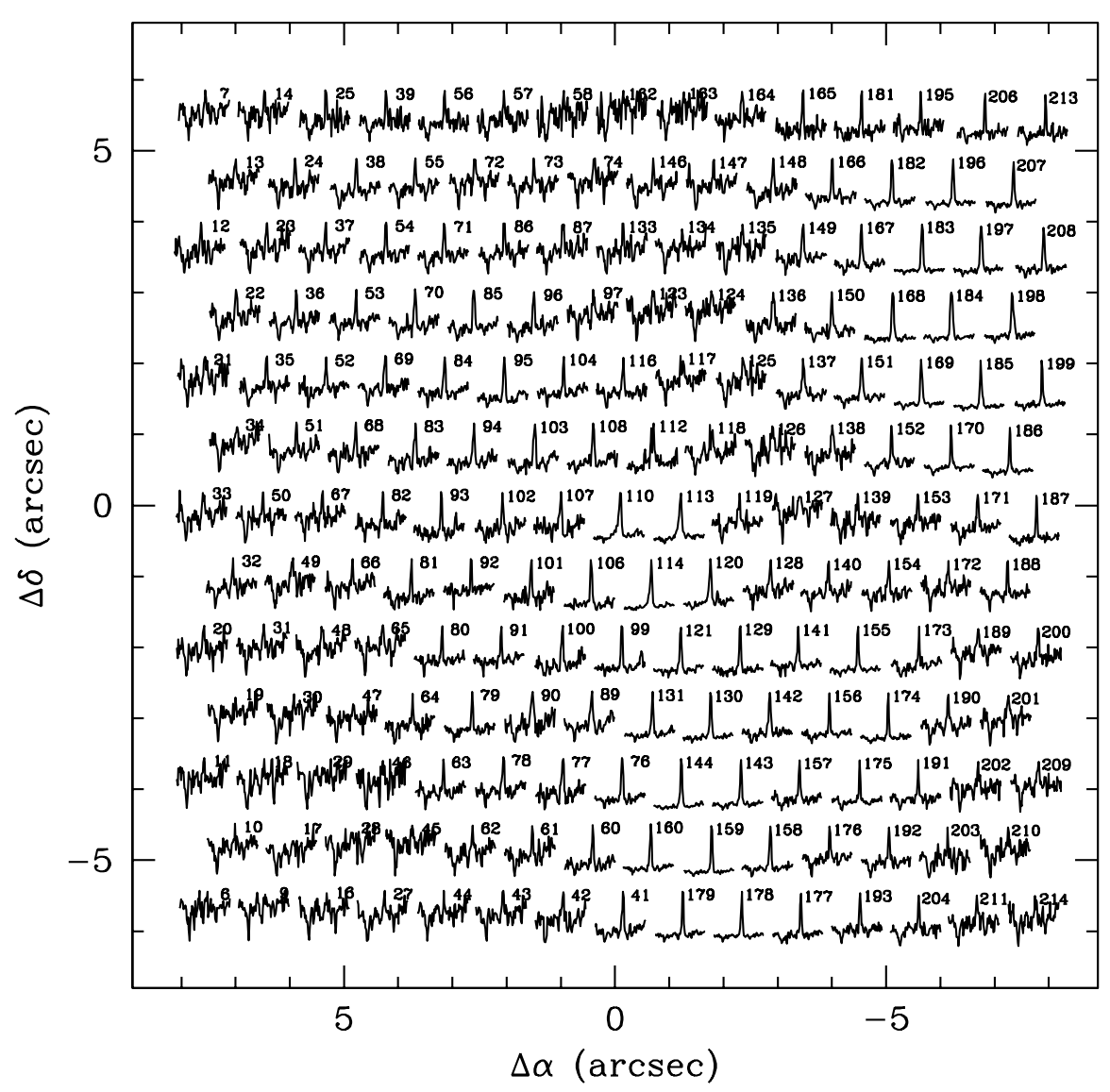

Fig. 2. b) Spectra in the circumnuclear region of NGC 2992 in the ranges: 4950-5080 $\AA$, including $\mathrm{H} \beta$.

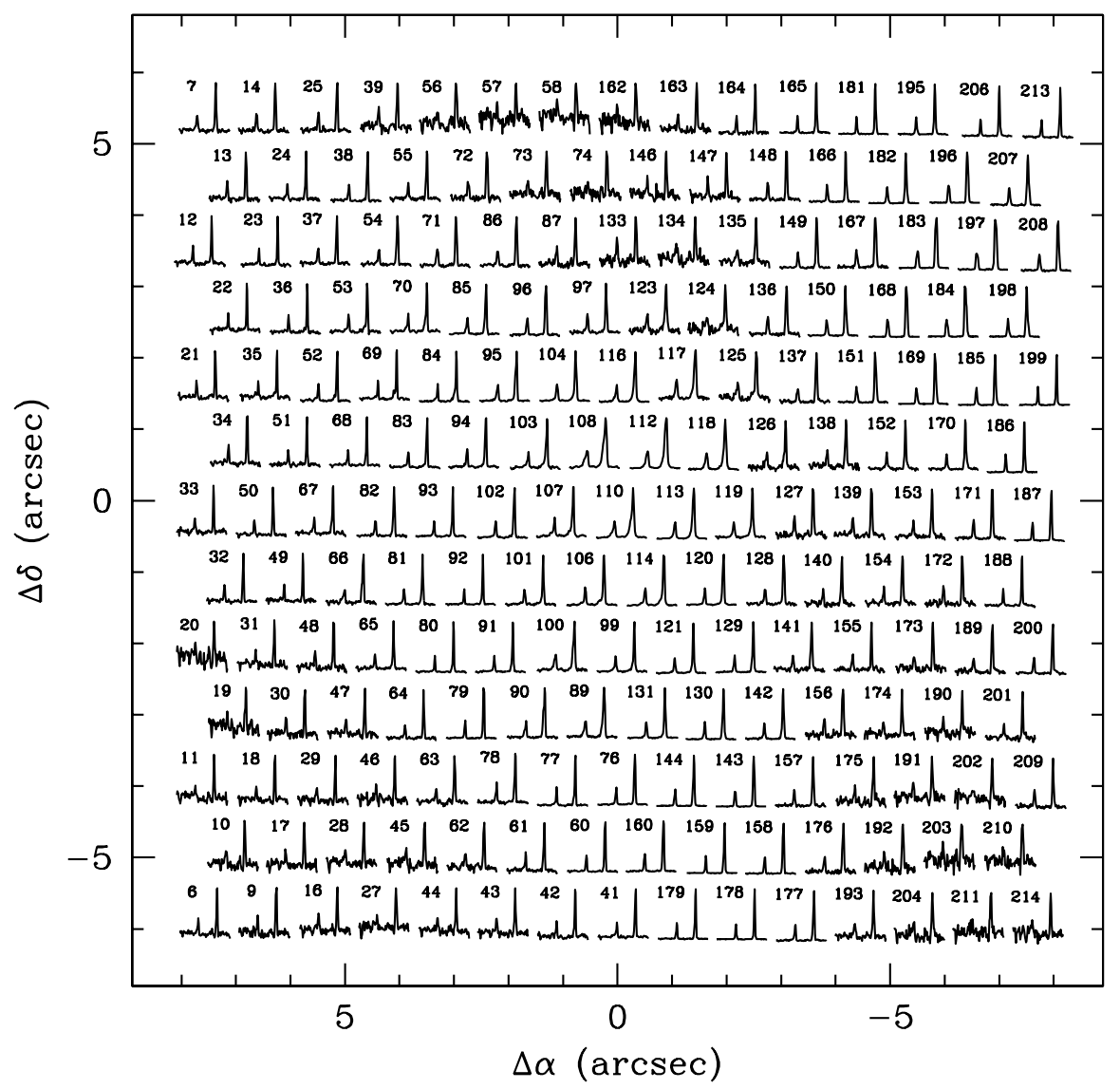

Fig. 2. c) $4950-5080 \AA$, including [O III $] \lambda 4959$ and [O III $] \lambda 5007$. 


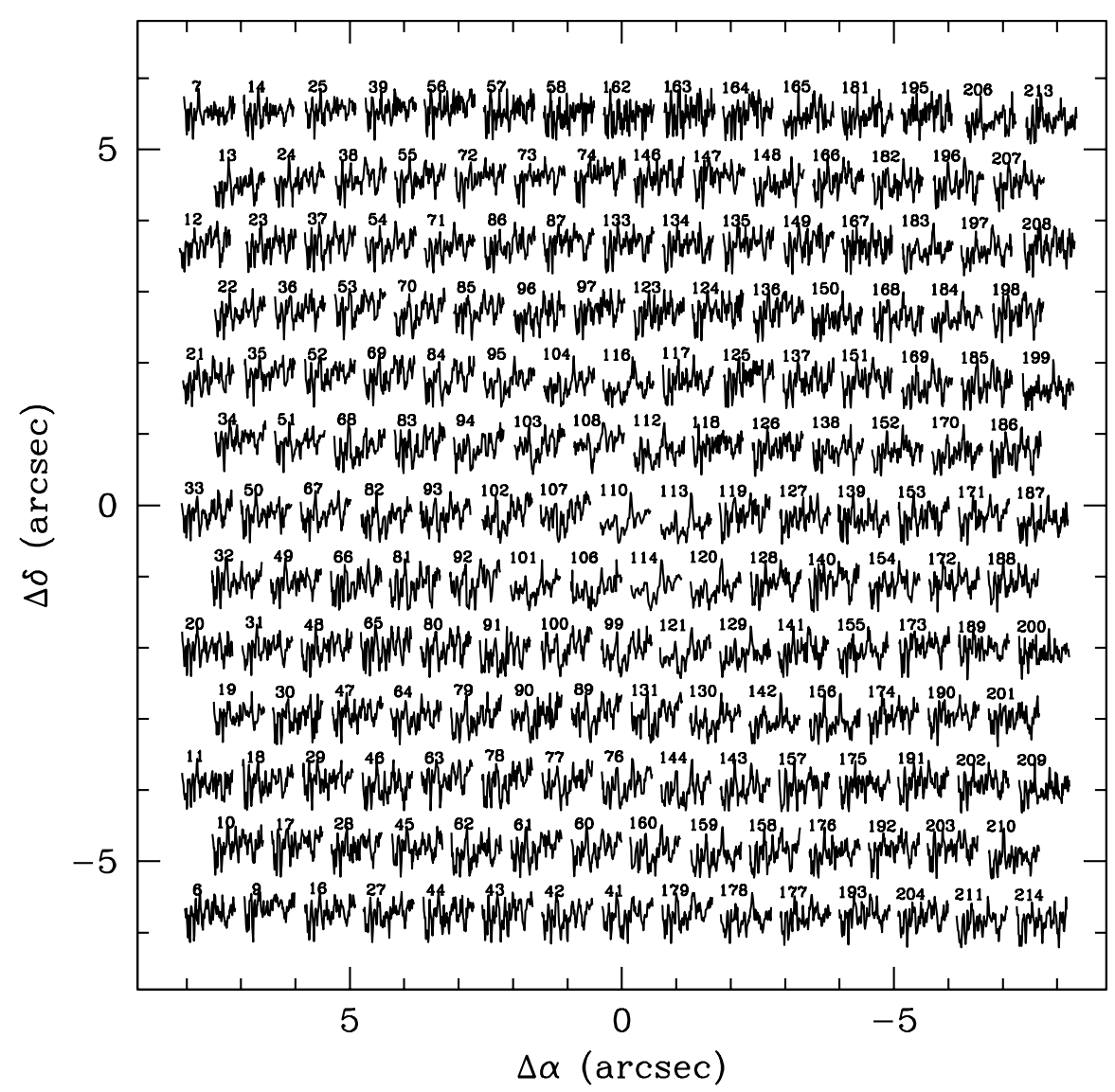

Fig. 2. d) $5160-5291 \AA$, including the absorption lines of $\mathrm{Mg} \mathrm{I} \lambda 5167,5172,5183$.

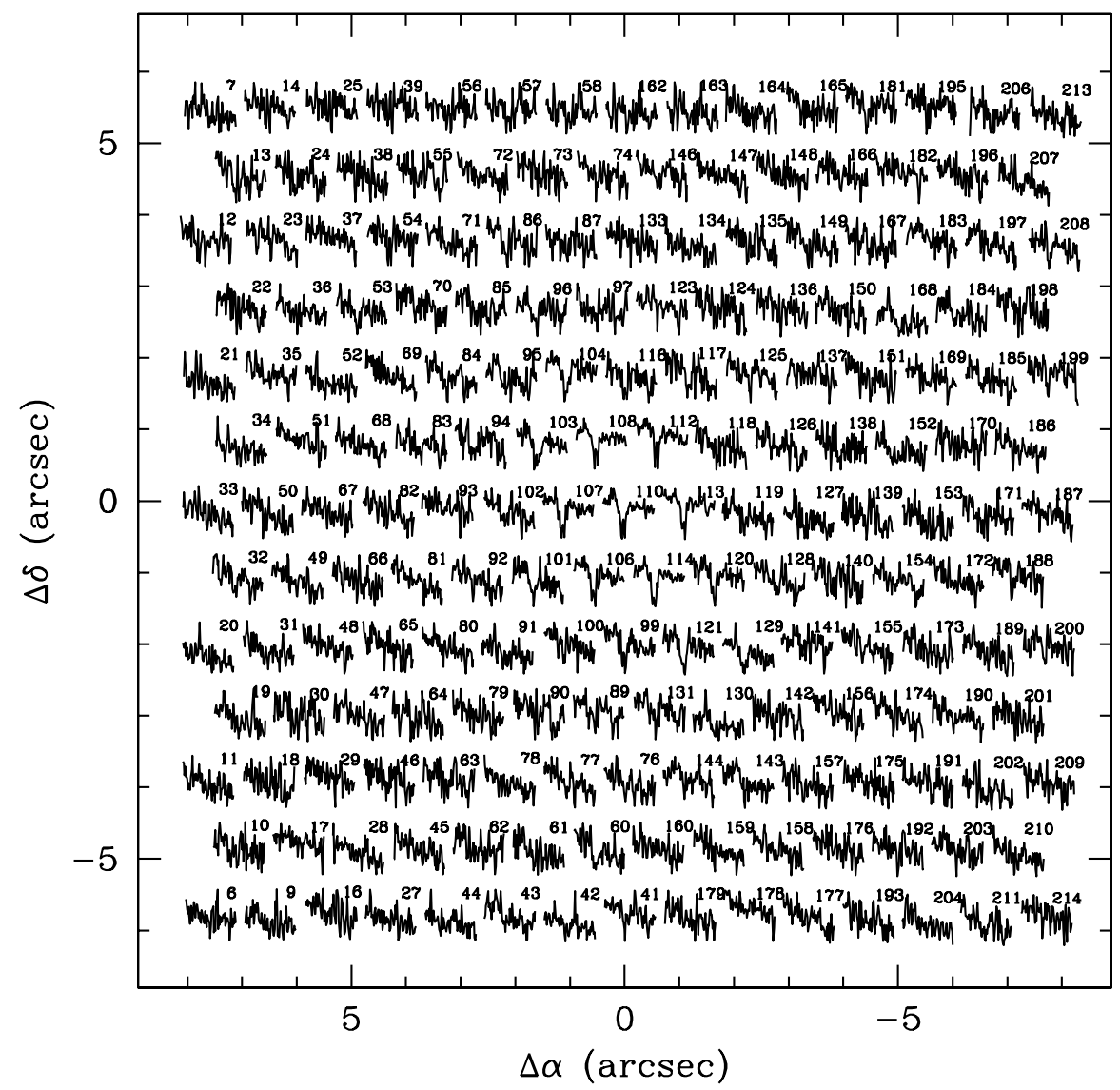

Fig. 2. e) 5900-6000 ̊, including the absorption lines of Na I $\lambda 5889,5895$. 


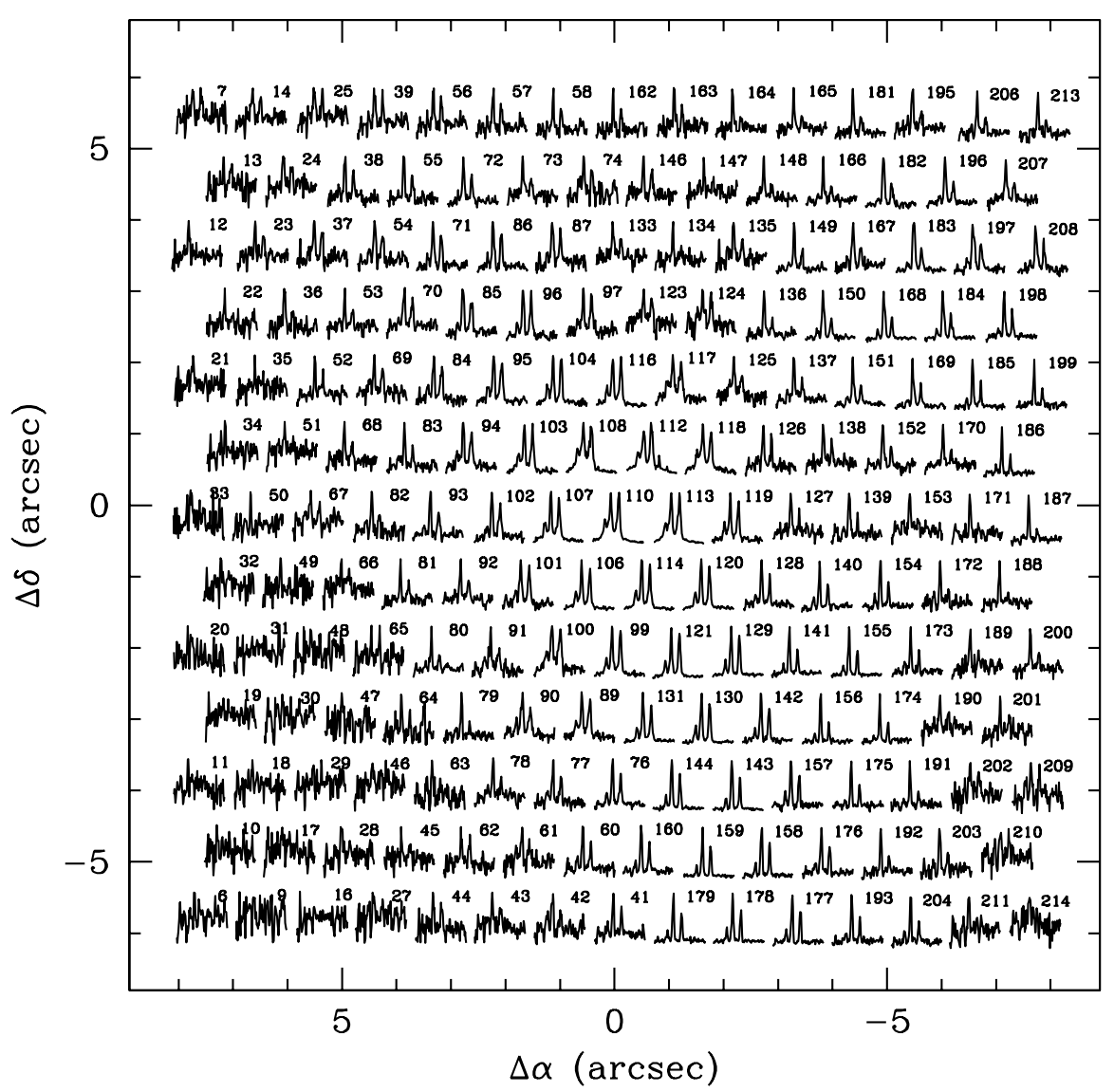

Fig. 2. f) $6569-6697 \AA$, including $\mathrm{H} \alpha \&[\mathrm{~N} \mathrm{II}] \lambda 6548,6584$.

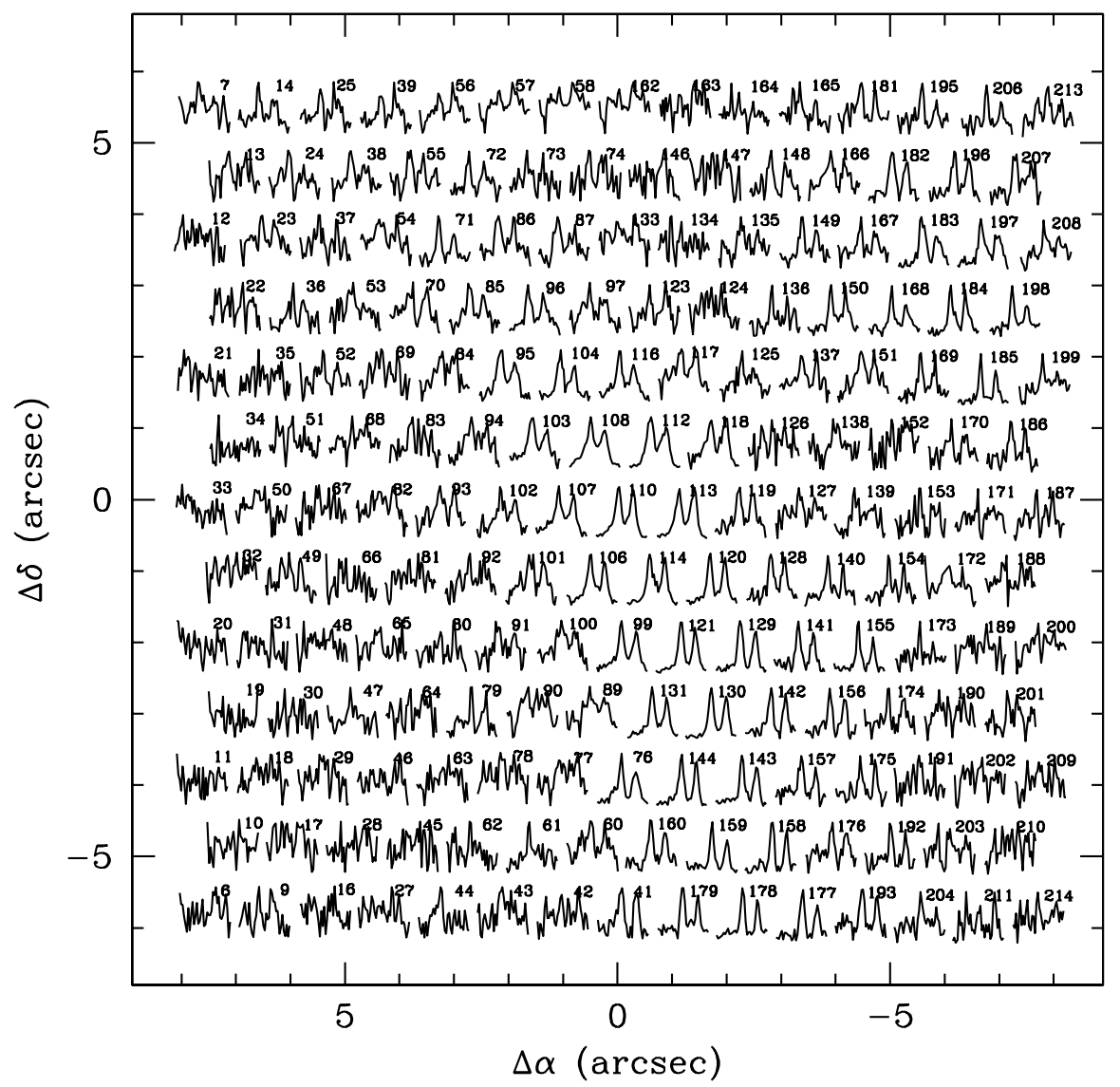

Fig. 2. g) 6747-6797 $\AA$, includes the emission lines of [S II] $\lambda 6716$ and [S II ] 26731 . 

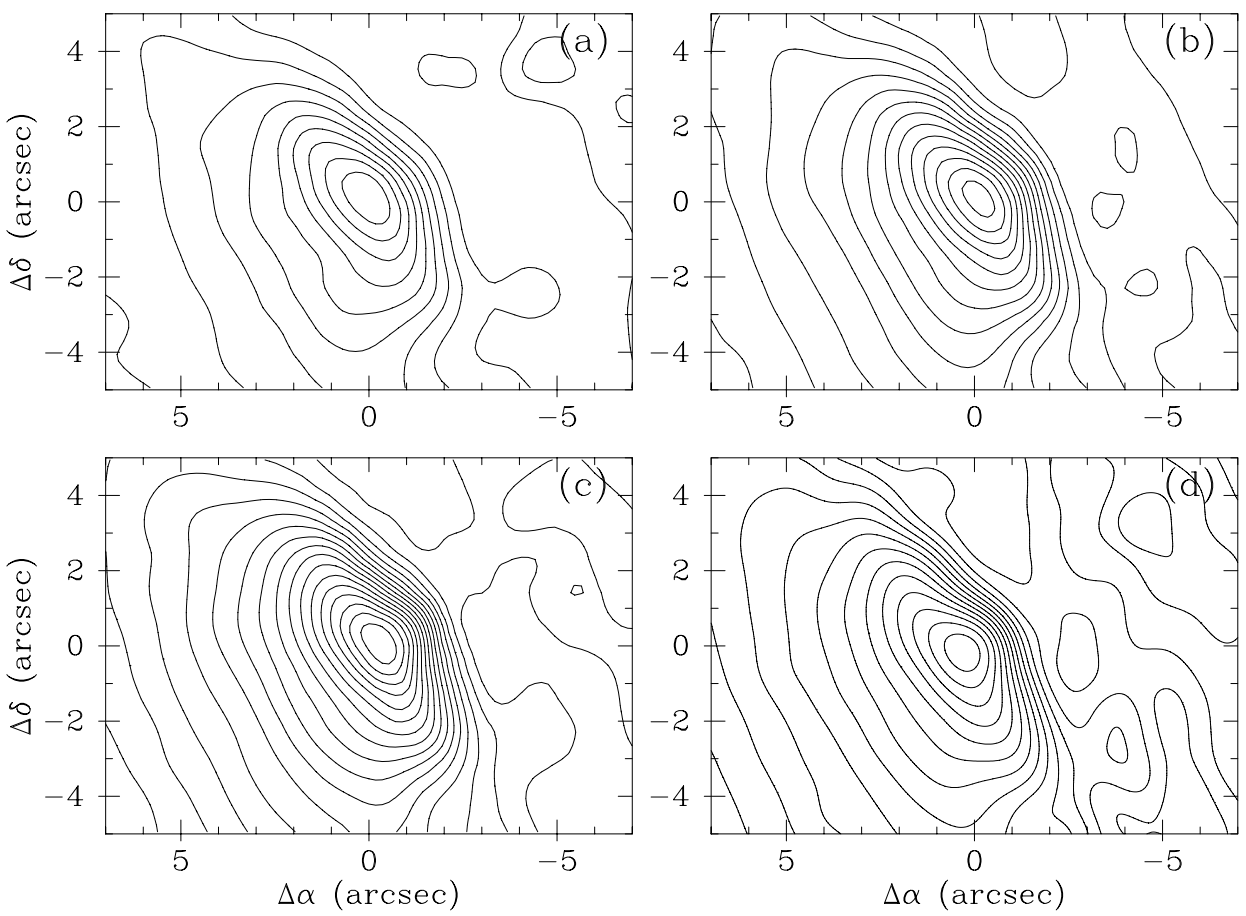

Fig. 3. Two-dimensional distribution of the continua in the spectral ranges a) 4500-4550 $\AA$, b) $5100-5150 \AA$, c) $5750-5800 \AA$. In these maps, and hereafter, north is up and east is left, as usual. d) corresponds to an HST (WFPC2) image in the F606W filter (centred on $5957 \AA$, and bandwidth $1508 \AA$ ) after convolution with a Gaussian ( $\sigma \sim$ mean seeing). This image is saturated in the continuum peak.

\subsection{Stellar velocity field}

In order to improve the signal-to-noise ratio, the spectra closer than $1^{\prime \prime} 2$ were added for each fibre position. This is almost equivalent to having hexagonal apertures $1.6^{\prime \prime}$ in radius at any position of our original fibres (superposed apertures) (see García-Lorenzo et al. 2000 for a non-smoothed velocity map). The mean stellar radial velocities were then obtained by applying the crosscorrelation technique in the range 5140-5260 $\AA$, which includes the Mg I $b$ lines. As a template we have used the solar $\mathrm{Mg} \mathrm{I} b$ scattered from moonlight, which are present in our spectra too. The cross-correlation was evaluated with the XCORR command of the DIPSO package by subtracting and dividing each spectrum by a properly fitted continuum in order to minimize edge effects. Figure 4 shows the stellar velocity field obtained in this way. Considering as independent sources of error those due to the wavelength calibration $\left(\sim 6 \mathrm{~km} \mathrm{~s}^{-1}\right)$, the template $\left(\sim 6 \mathrm{~km} \mathrm{~s}^{-1}\right)$, and the finite $S / N\left(\sim 15 \mathrm{~km} \mathrm{~s}^{-1}\right)$, conservative final uncertainties of about $20 \mathrm{~km} \mathrm{~s}^{-1}$ are expected. The velocity of the optical nucleus derived from this map is $2300 \pm 20 \mathrm{~km} \mathrm{~s}^{-1}$. This value is in agreement with the systemic velocity $\left(V_{\text {sys }}=2330 \mathrm{~km} \mathrm{~s}^{-1}\right)$ determined from Ca II absorption lines in the near infrared by Nelson \& Whittle (1995).

In the velocity field a flat structure aligned at PA $30^{\circ}$ stands out. It seems to be a rotating disc seen almost edge on with the major kinematic axis aligned with the inner photometric axis. The flat structure becomes narrower at 3 arcsec (see top of Fig. 4). Such behaviour could be explained by a warped disc whose inclination increases

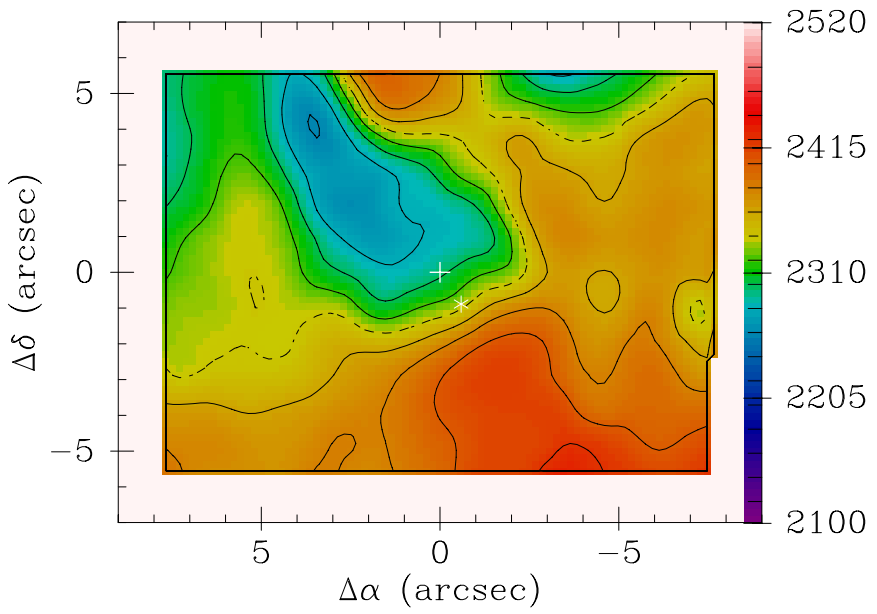

Fig. 4. Stellar velocity field in the central region of NGC 2992 inferred from $\mathrm{Mg} \mathrm{I} b$ lines. The isovelocity lines span from 2100 to $2480 \mathrm{~km} \mathrm{~s}^{-1}$ with steps of $20 \mathrm{~km} \mathrm{~s}^{-1}$. The dashed isovelocity line corresponds to the systemic velocity of NGC 2992 (2330 $\mathrm{km} \mathrm{s}^{-1}$, Nelson \& Whittle 1995). The white cross and asterisk mark the optical nucleus position and stellar the kinematic centre, respectively.

from the center to the outer regions. Unfortunately, we cannot properly fit a kinematical model to derive the radial variation of the inclination of NGC 2992, as this is an edge-on galaxy and our spatial coverage is small.

Outside the disc, the isovelocity lines bend to connect with the more slowly rotating bulge. The kinematic minor axis seems to be also bent as if the bulge and disc 
rotational axes were not coincident. However, our small spatial coverage does not allow us to determine properly the direction of the minor axis of the bulge.

To derive the kinematic centre, we took the "differential" of the stellar velocity field using the IDIFF command in the FIGARO package (Starlink Software Documentation: http://star-www.rl.ac.uk). This process creates a new image where each pixel is the average absolute difference between the corresponding pixel value in the velocity field and its immediate neighbourhood. This emphasizes the regions where the velocities are changing more rapidly. The location of the kinematic centre (K hereafter) so obtained was $\Delta \alpha=0.6 \pm 0.2 \mathrm{~W}$ and $\Delta \delta=1.0 \pm 0.2 \mathrm{~S}$ with respect to the optical nucleus. The radial velocity of $\mathrm{K}$ is $V_{\mathrm{r}}(\mathrm{K})=2341 \pm 20 \mathrm{~km} \mathrm{~s}^{-1}$, which is also in good agreement with previous measurements of the systemic velocity using stellar features (Nelson \& Whittle 1995). Notice that this kinematic centre has been inferred in the region of highest velocity gradient corresponding to the disc. Consequently, it should be regarded exclusively as the rotation centre of this structure.

Velocity dispersions have been estimated from the width of the cross-correlation function, but the twodimensional distribution shows a patchy structure, probably due to the uncertainties associated with and a relatively constant value over the observed region. We have determined a mean value of $167 \pm 5 \mathrm{kms}^{-1}$ and $142 \pm 8 \mathrm{~km} \mathrm{~s}^{-1}$ for the optical nucleus and $\mathrm{K}$, respectively. These values are in good agreement with previous determinations of velocity dispersions $\left(\sigma_{*}=158 \pm 13 \mathrm{~km} \mathrm{~s}^{-1}\right.$; Nelson \& Whittle 1995, and references therein). We have obtained a radial profile of stellar velocity dispersions by interpolating elliptical rings (centred on the optical nucleus and also on K) to its 2D patchy structure (Fig. 5).

\section{5. lonized gas}

\subsection{Ionized gas distribution and ionization}

Figure 6 shows line intensity maps obtained fitting a single-Gaussian profile to each emission line. These maps are presented on a logarithmic stretch in order to bring out the fainter regions. The emission is bipolar presenting two main regions ( $\mathrm{A}$ and $\mathrm{B}$ ) separated by the dust lane, whose emission peaks are located $\sim 0 . .56$ and $\sim 60^{\prime \prime} 5$ toward the NW from the optical nucleus, respectively (notice that A and $\mathrm{B}$ are not aligned with the disc kinematic centre). An additional peak (C) appears $\sim 3^{\prime \prime} .9 \mathrm{SW}$. A knot of emission (D) is detected in the dust lane at $\sim 5^{\prime \prime} \mathrm{SW}$, which is probably associated with an $\mathrm{H}$ II region. The [O III] emission map also shows an extended arc-shaped region (E) toward the SE, this being a weak feature in the other maps.

Narrow-band images of NGC 2992 have been interpreted in terms of an extensive biconical ENLR emerging almost perpendicularly from the plane of the galaxy (see Allen et al. 1999, hereafter A99). According to this view, the nucleus should be placed at the apex of this

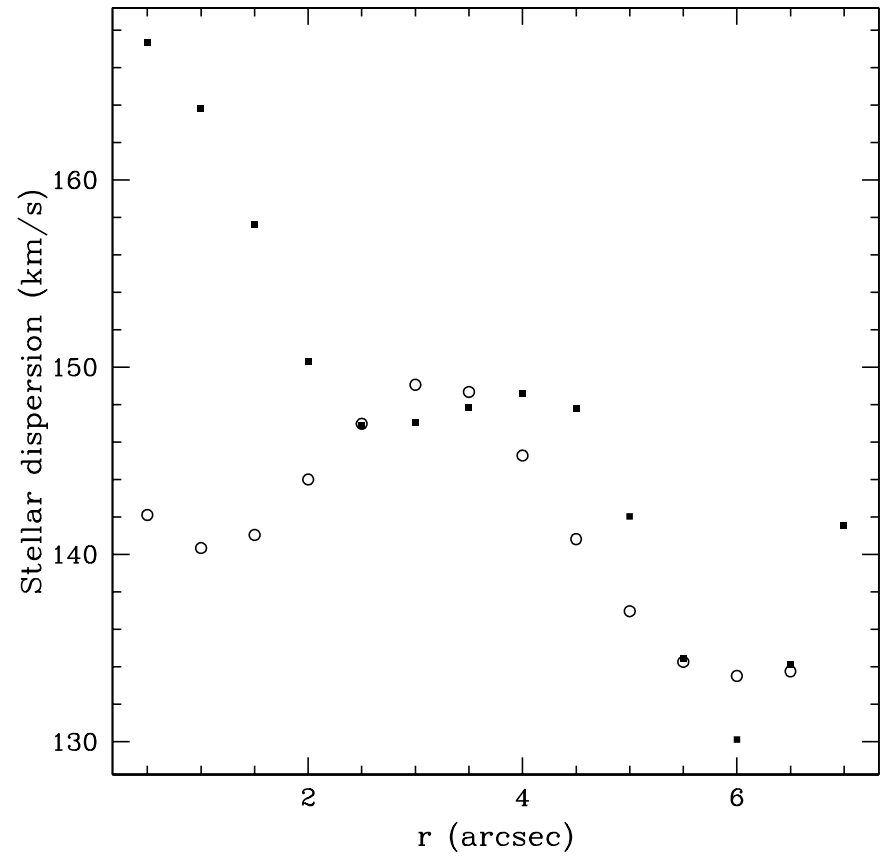

Fig. 5. Radial distribution of velocity dispertion for the stellar component. The rms of the individual average values is $\sim \pm 15 \mathrm{~km} \mathrm{~s}^{-1}$. Filled squares and open circles are values taking the centre in the optical nucleus and $\mathrm{K}$, respectively.

biconical structure. The well-defined bipolar shape of our [O III] intensity map (Fig. 6) allows us to place the apex at $\Delta \alpha=2$." $4 \mathrm{~W}$ and $\Delta \delta=1$ ". $1 \mathrm{~N}$ from the optical nucleus with an uncertainty of $\pm 0^{\prime \prime} 1$ in both directions. We have determined an orientation almost perpendicular $\left(\sim 125^{\circ}\right)$ to the photometric major axis in the inner region $\left(\sim 35^{\circ}\right)$, and an opening angle of $\sim 130^{\circ}$ for the bipolar structure. These values are in excellent agreement with those found by VSM01.

The $[\mathrm{O}$ III $] / \mathrm{H} \beta$ map (Fig. 6) also depicts the biconical structure, showing a clear dichotomy between the dust lane region (low values) and elsewhere. High values (high ionization) are found in three distinct zones: i) around the intensity peak (A), ii) in the boundaries of region $B$ and iii) in a region towards south-east $(\mathrm{F})$, near the arc-shaped emission ( $\mathrm{E})$. The high $[\mathrm{O} \mathrm{III}] / \mathrm{H} \beta$ value in region $\mathrm{F}$ can be (partially) explained by the presence of $\mathrm{H} \beta$ in absorption there (A99). The $\mathrm{B}$ and $\mathrm{E}$ (or F) regions exhibit different [NII] $/ \mathrm{H} \alpha$ ratios (see diagnostic diagrams in Fig. 7). The average $[\mathrm{N} \mathrm{II}] / \mathrm{H} \alpha$ ratios for regions $\mathrm{B}$ and $\mathrm{E}$ (or $\mathrm{F}$ ) are, respectively, $0.42 \pm 0.05$ and $0.62 \pm 0.12$ (or $0.61 \pm 0.08$ ), whereas the mean value for region $\mathrm{D}$ in the dust lane is $0.42 \pm 0.03$. Although the stellar $\mathrm{H} \alpha$ absorption is larger in the $\mathrm{E}$ (or $\mathrm{F}$ ) region than in $\mathrm{B}$, the excess is not large enough to explain the differences in the $[\mathrm{N} \mathrm{II}] / \mathrm{H} \alpha$ ratio. Consequently, a real difference in the excitation conditions should exist between the E (or F) and B regions (A99). The same result is inferred from the $[\mathrm{S} \mathrm{II}] / \mathrm{H} \alpha$ ratio, the mean values being $0.60 \pm 0.11,0.33 \pm 0.05$, and $0.50 \pm$ 0.11 , for regions $\mathrm{E}, \mathrm{D}$ and $\mathrm{B}$, respectively. We do not have any emission from $[\mathrm{S}$ II $]$ in the $\mathrm{F}$ region. We have also 
Table 1. Logarithmic ratio for different regions.

\begin{tabular}{|c|c|c|c|c|c|c|}
\hline Region & {$[\mathrm{O}$ III $] \lambda 5007 / \mathrm{H} \beta$} & $\mathrm{rms}$ & {$[\mathrm{N}$ II $] \lambda 6584 / \mathrm{H} \alpha$} & $\mathrm{rms}$ & {$[\mathrm{S} \mathrm{II}] \lambda 6716+6730 / \mathrm{H} \alpha$} & $\mathrm{rms}$ \\
\hline $\mathrm{A}$ & 1.11 & 0.07 & 0.00 & 0.02 & -0.21 & 0.06 \\
\hline B & 1.01 & 0.06 & -0.37 & 0.05 & -0.30 & 0.08 \\
\hline $\mathrm{C}$ & 0.93 & 0.05 & -0.16 & 0.04 & -0.22 & 0.04 \\
\hline $\mathrm{D}$ & 0.11 & 0.10 & -0.37 & 0.03 & -0.48 & 0.07 \\
\hline $\mathrm{E}$ & 1.11 & 0.04 & -0.22 & 0.09 & -0.23 & 0.08 \\
\hline $\mathrm{F}$ & 1.22 & 0.12 & -0.22 & 0.06 & & \\
\hline $\mathrm{G}$ & 0.67 & 0.25 & -0.22 & 0.06 & -0.22 & 0.13 \\
\hline
\end{tabular}

included a region, $\mathrm{G}$, to the north of the optical nucleus in Fig. 6. This region is not relevant at this point but will be discussed in Sect. 6.3. Table 1 gives the mean values ratio for each region and its associated rms.

Both diagrams show that ratios mainly fall within the Seyfert galaxy region. Our diagrams are in good agreement with those in A99, who performed a comprehensive study of the physical conditions in the circumnuclear region of NGC 2992, concluding that a shock + precursor model is most consistent with the observed line ratios. This model considers that in the high-temperature cooling zone behind the shock front, high velocity shock can generate strong UV photons. This radiation is then available to produce a highly ionized precursor $\mathrm{H}$ II region, emitting an NLR-like spectrum (Dopita \& Sutherland 1995).

\subsection{Mean velocity field of the ionized gas}

The individual mean radial velocities of the ionized gas were obtained by cross-correlating spectra. This was done in four different spectral ranges, including the $\mathrm{H} \beta$, [O III] $\lambda \lambda 4959,5007, \mathrm{H} \alpha+[\mathrm{N} \mathrm{II}] \lambda \lambda 6548,6584$, and $[\mathrm{S}$ II] $\lambda 6716,6730$ lines. As a template, we have selected the observed spectrum from fibre 179 (see Figs. 2b,c,f and g) which has well-defined and symmetric line profiles and also a relatively high $S / N$ in all cases. The absolute velocities were derived by Gaussian fitting to the emission lines of the template. Figure 8 shows the mean velocity fields obtained.

The stellar kinematic centre $(\mathrm{K})$ seems also to be a good kinematic centre for the ionized gas. Derived velocities for $\mathrm{K}$ are $2356 \pm 20 \mathrm{~km} \mathrm{~s}^{-1}, 2328 \pm 19 \mathrm{~km} \mathrm{~s}^{-1}$, $2358 \pm 15 \mathrm{~km} \mathrm{~s}^{-1}$ and $2371 \pm 20 \mathrm{~km} \mathrm{~s}^{-1}$ for the same lines, respectively. Therefore, we have considered a mean value of $2353 \pm 18 \mathrm{~km} \mathrm{~s}^{-1}$ for the heliocentric velocity of $\mathrm{K}$. This value agrees, within the errors, with previously reported measures (see VSM01 and references therein). It also agrees with the obtained stellar velocity value for $\mathrm{K}$ (see Sect. 4.2). The heliocentric velocities derived for the optical nucleus are $2265 \pm 19 \mathrm{~km} \mathrm{~s}^{-1}$, $2204 \pm 23 \mathrm{~km} \mathrm{~s}^{-1}, 2301 \pm 15 \mathrm{~km} \mathrm{~s}^{-1}$ and $2312 \pm 15 \mathrm{~km} \mathrm{~s}^{-1}$ from $\mathrm{H} \beta,[\mathrm{O}$ III] $\lambda \lambda 4959,5007, \mathrm{H} \alpha+[\mathrm{N} \mathrm{II}] \lambda \lambda 6548,6584$, and $[\mathrm{S} \mathrm{II}] \lambda 6716,6730$, respectively (in principle, the differences are surprisingly large but notice that the optical nucleus has no special kinematic meaning). The velocity maps of the ionized gas in Fig. 8 show remarkable agreement.

The most relevant differences in the ionized gas with respect to the stellar velocity field are: i) the presence of a red region in the NW corner, ii) the strong blueshift around the optical nucleus, and iii) the existence of a red protrusion at the SE $\left(\Delta \alpha=2^{\prime \prime}, \Delta \delta=-4^{\prime \prime}\right)$. The red region to the NW and the blueshifted region around the optical nucleus are almost aligned with the apex of the bipolar emission-line structure (Sect. 5.1). This is an indication of the presence of an outflowing component, similar to what has been found in other Seyfert galaxies (see, for example, Arribas et al. 1996). The presence of an outflow in the inner regions of NGC 2992 has also been reported by previous authors (see, for instance, VSM01). Comparing the $\mathrm{H} \beta$ and the $\left[\begin{array}{ll}\mathrm{O} & \mathrm{II}\end{array}\right]$ maps, we found that the innermost region surrounding the nucleus appears to be more blueshifted in the [O III] map. This suggests that a highionization component associated with the AGN has a distinct kinematic behaviour, probably associated with the outflow. Finally, the red protrusion, which extends over about $600 \mathrm{pc}$, is in positional agreement with region $\mathrm{E}$ of high ionization found to the SE of the optical nucleus (Fig. 6).

\section{Discussion}

\subsection{Outflowing gas}

To analyse the ionized gas kinematics, we take as a reference the stellar component. In particular, the relative velocity shifts between the gas and the stars can give us a clear idea of the effects of the interaction and/or the AGN on the gas. Figure 9 presents the relative shift map.

The blue and red velocities associated with the nucleus (region A) and the NW structure (region B), respectively, can be well interpreted by the outflow previously reported by other authors (see e.g. VSM01). In fact these two regions are located rather symmetrically on both sides of the dust lane in the intensity line map (Fig. 9b). Moreover, the apex of the bipolar [O III] emission (Sect. 5.1) seems to be a good location for the origin of this outflow. In particular, a simple rotation + outflow model, including a constant radial velocity of $150 \mathrm{~km} \mathrm{~s}^{-1}$, is a good approximation 

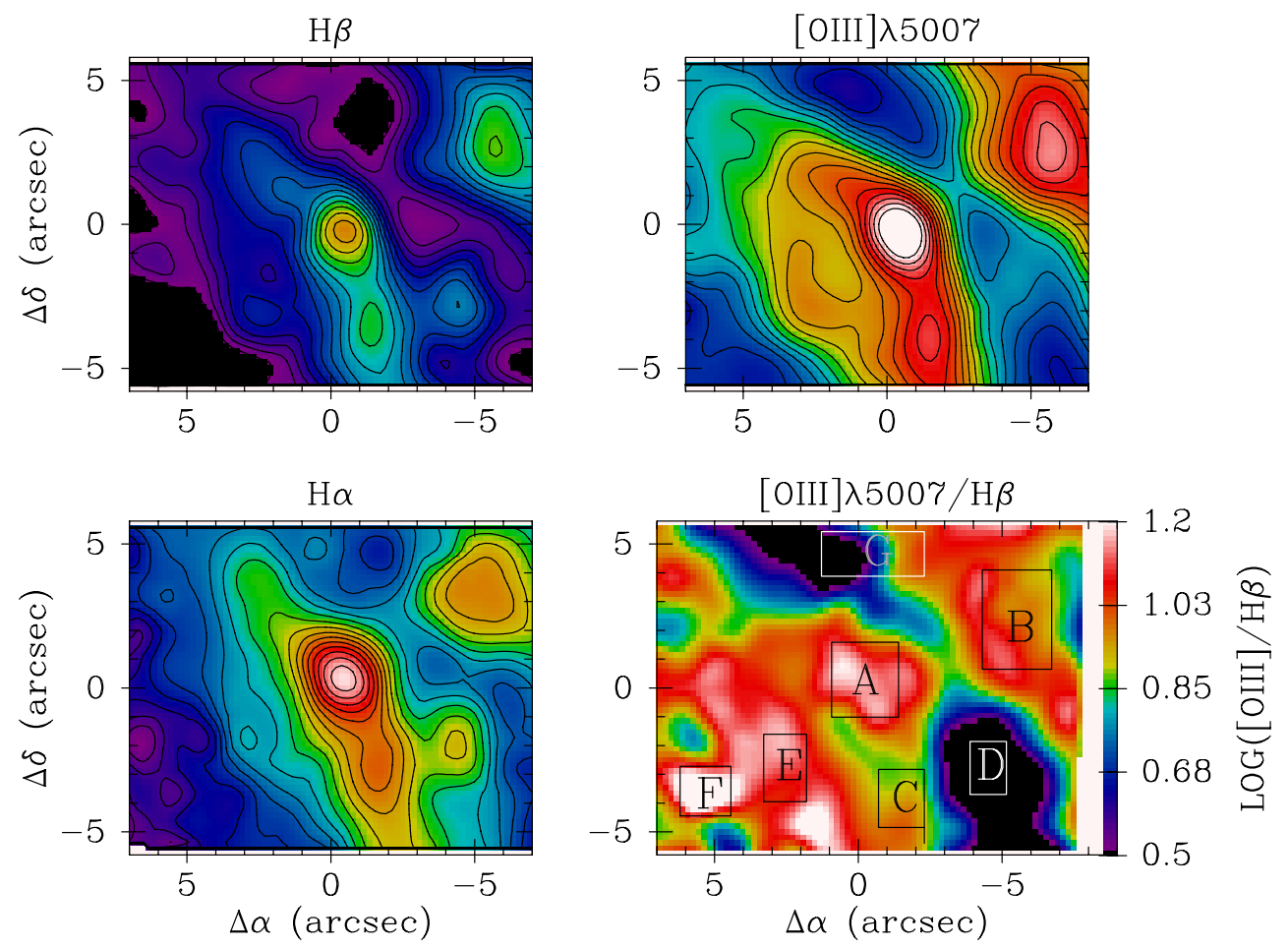

Fig. 6. Integrated line intensity maps of the indicated emission line derived by fitting a single Gaussian to each emission line. The $[\mathrm{O}$ III $] \lambda 5007 / \mathrm{H} \beta 2 \mathrm{D}$ ratio is plotted in the fourth box. Emission peaks regions have been indicated in this plot (see text).
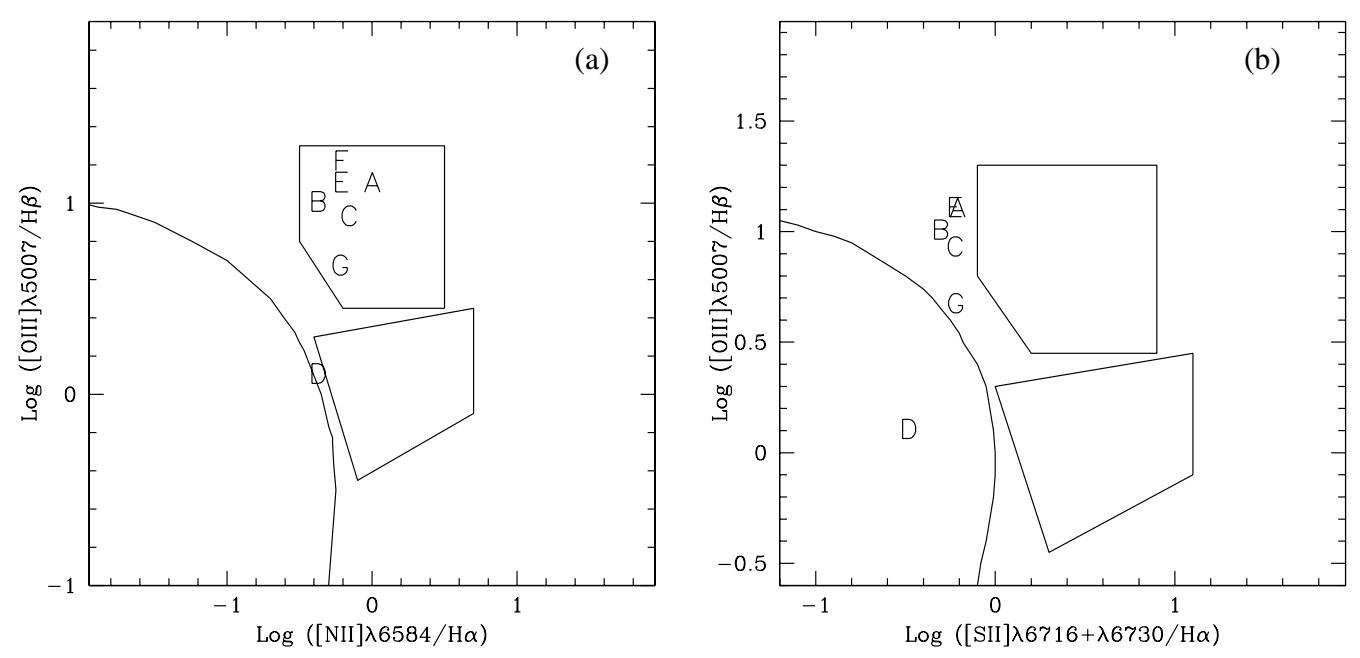

Fig. 7. Diagnostic diagrams for the different labelled regions. Lines indicate limits of photoionization by H II regions, shocks or AGN (see, for example, Veilleux \& Osterbrock 1987).

for NGC 2992 kinematics (MBDP98), although recent measurements suggest a non-constant outflow velocity (VSM01). The radial velocities derived from Fig. 9 are $\sim \pm 90 \mathrm{~km} \mathrm{~s}^{-1}$ and are in good agreement with the results of VSM01. This kinematic/morphological pattern is reminiscent of what is found in other Seyfert 2 galaxies such as NGC 5728 (Arribas \& Mediavilla 1993) and NGC 1068 (García-Lorenzo et al. 1999).

Three possible models of the origin of the outflow are discussed in VSM01. Starburst-driven wind and collimated radio jets are ruled out due to the absence of a powerful nuclear starburst and diffuse radio morphology in NGC 2992. The shock + precusor model (Dopita \& Sutherland 1995) seems to be the most reasonable scenario for the origin of the radiation field when checked against both the physical conditions (A99) and outflow velocities (VSM01).

However, Fig. 9 indicates that a simple two-component model (regular rotation + outflow) cannot fully explain the ionized gas velocity field in NGC 2992. In fact, even excluding the velocity shifts associated with the outflow, Fig. 9 presents significant kinematic structures. 

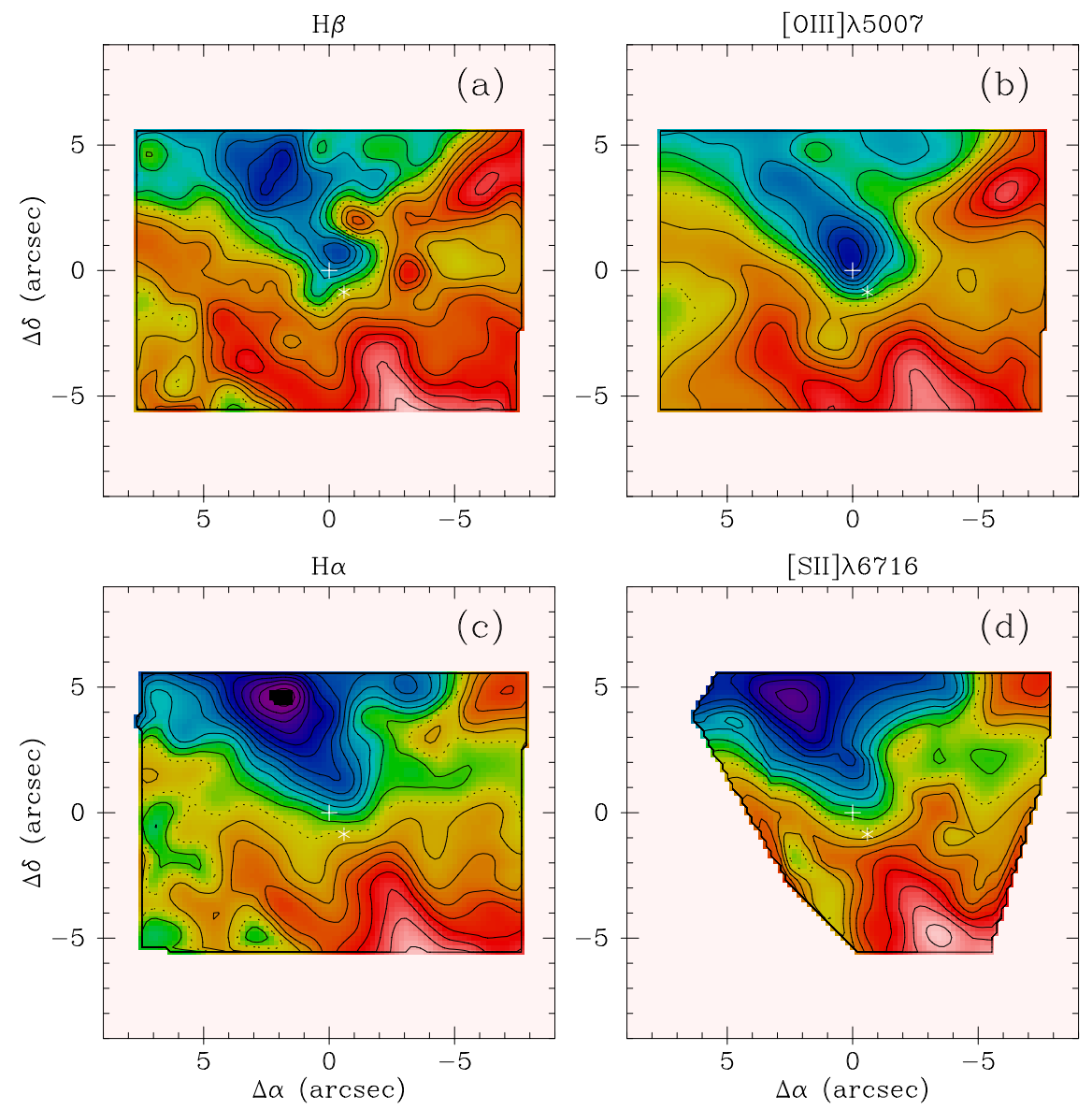

Fig. 8. Velocity fields of the ionized gas in the central region of NGC 2992 inferred from cross-correlation in the ranges a)

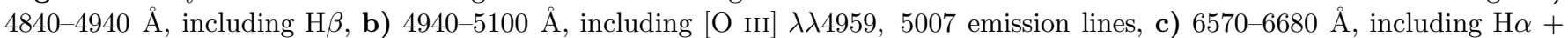
[N II] $\lambda \lambda 6548,6584$, and d) 6720-6820 , which includes [S II] $\lambda \lambda 6716,6731$. The white cross marks the optical nucleus location. Isovelocity lines expand from 2100 to $2500 \mathrm{~km} \mathrm{~s}^{-1}$ in steps of $20 \mathrm{~km} \mathrm{~s}^{-1}$. The systemic velocity $\left(2340 \mathrm{~km} \mathrm{~s}^{-1}\right)$ is indicated by a dashed line.

\subsection{Presence of a third gaseous component: Optical correlation with the radio structure?}

The "residual map" of velocities (Fig. 9) shows a (semicircular) arc of redshifted velocities to the south-east (corresponding to the red protrusion of the original ionized gas velocity maps discussed in Sect. 5.2). This arc is coincident with the arc of emission in the ionized gas distribution (Fig. 9b), as well as with the high-ionization region to the SE (region E). To the North, the residual map also shows a blueshifted region extended to the west (region G, hereafter, and marked in Fig. 6d). The mean velocities for the redshifted and blueshifted "arcs" are $64 \pm 18 \mathrm{~km} \mathrm{~s}^{-1}$, and $-79 \pm 28 \mathrm{~km} \mathrm{~s}^{-1}$, respectively.

As at present, there were no obvious correlations between the radio structure (figure-of-8) and any optical structure in NGC 2992. However, both red and blue structures in the "residual map" resemble the loops of the figure of 8-shaped region detected in the $6 \mathrm{~cm}$ radio emission (Ulvestad \& Wilson 1984). In spite of uncertain alignment of the radio and optical observations, the superposition of the radio figure 8 (Fig. 9a) on the "residual" map, suggests that red- and blueshift velocities could be closely related to the radio emission.

On the one hand, the weak feature presents in our [O III] intensity map (weaker for other lines), the SE high ionization region, as well as the red arc of the "residual velocities map" seem to establish a clear correspondence with the south portion of the figure-of-8-shape radio structure. On the other hand, because the NW side of the galaxy is the nearest, we would expect a brighter blue arc. However, the dust lane crossing the galaxy strongly hides the emission coming from this region. In fact, spectra from this region have low $S / N$, and reddening is much higher along the dust lane than in other parts of the ENLR (A99). In spite of this, the "residual" velocities map seems to establish a correlation between region $\mathrm{G}$ and the upper side of the radio figure-of-eight structure.

In this scenario, an overpressure relativistic plasma is expanding and compressing the gas in its path. The interaction of the radio ejecta and the ambient medium could also produce ionizing radiation (Dopita \& Sutherland 1995), which well explains the high ionization of regions $\mathrm{E}$ and $\mathrm{G}$. 

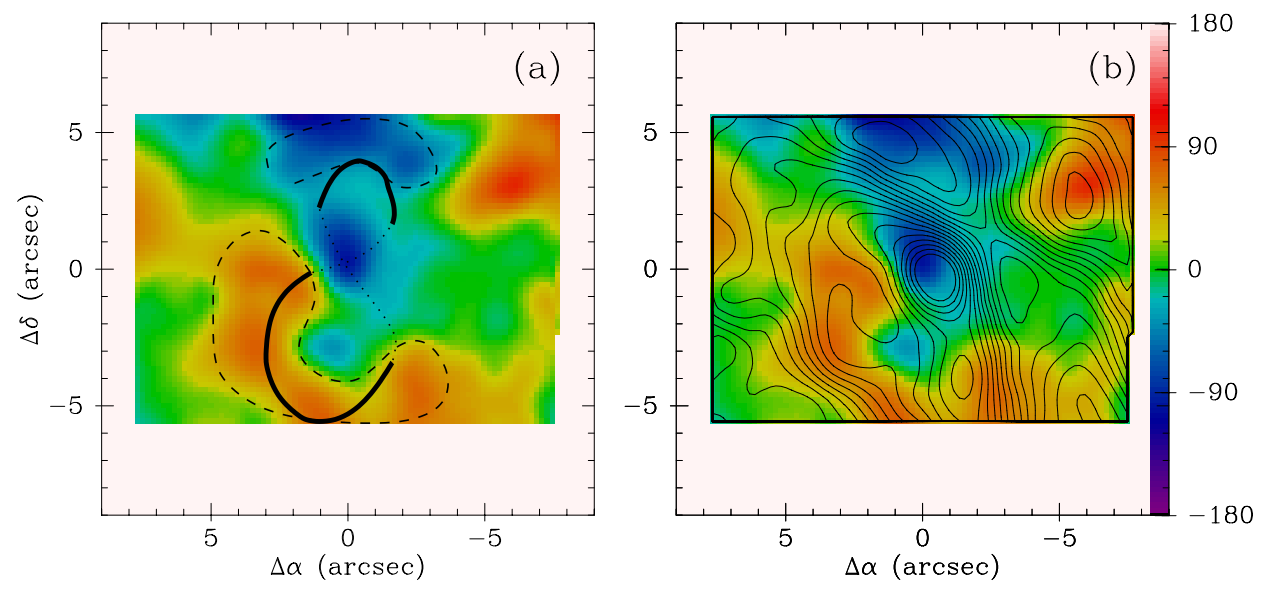

Fig. 9. Residual map of velocities. This map has been obtained by subtracting the stellar velocity field from the ionized gas velocity field of [O III]. We have superposed: a) full black line: the boundaries of the $6 \mathrm{~cm}$ radio morphology; dotted line: the figure-of-eight-shaped structure of the $6 \mathrm{~cm}$ radio emission centred on the optical nucleus; dashed line: regions $\mathrm{E}$ and $\mathrm{G}$ (see text), and b) the intensity map of [O III].

If the red and blue arcs of the "residual map" are then really associated with the radio loops, shifted velocities would be indicative of an "inflow" or "outflow" in its boundaries. The actual loops of the figure-of- 8 seems to lie out of the galactic disk plane (Chapman et al. 2000, CMAH hereafter). However, there are no real constraints on the orientation of the loops. If the north-west portion of the loops is closer to us, shifted velocities indicate an "outflow". In contrast, if the south-east portion of the figureof-8 is closer to us, shifted velocities suggest an "inflow". The last option is completely beyond our understanding if the radio loops are expanding. In this picture, a more comprehensive view is one in which the radio morphology has a component that lies in the plane of the galaxy and accelerates the gas in its path. In fact, spectra of regions $\mathrm{E}$ and $\mathrm{G}$ are narrow and well defined with no evidence of double components or asymmetries in the emission-line profiles associated with those regions. This hypothesis is in good agreement with adaptive optics NIR imaging of NGC 2992 $(\mathrm{CMAH})$. CMAH suggest that the radio figure-of-8-shape morphology is due to two superimposed components, one associated with the galaxy disc, and another flowing out of the galaxy plane. Moreover, the radio component in the disc could be associated with starburst supernova remnants. This option could explain the low expansion velocity observed $\left(\sim \pm 70 \mathrm{kms}^{-1}\right)$, and the velocity dispersions obtained for regions $\mathrm{E}\left(\sigma \sim 65 \pm 25 \mathrm{~km} \mathrm{~s}^{-1}\right)$ and $\mathrm{G}\left(\sigma \sim 80 \pm 10 \mathrm{~km} \mathrm{~s}^{-1}\right)$. However, VSM01 did not find evidence for a starburst in this object.

If this interpretation is correct, none of the outflowing components of the ionized gas in the inner $(<2 \mathrm{kpc})$ region of NGC 2992 are directly affected by the interaction with NGC 2993. The departure of the ionized gas from the regular stellar behaviour can be explained only by the presence of the AGN.

\subsection{Location of the hidden nucleus}

Assuming that the west side of the galaxy is the nearest to us (MBDP98), the outflow should correspond to radial motions in a plane less inclined than the stellar disc. The biconical shape of the [O III] intensity map suggests that radial motion could occur within a cone, its axis being closely perpendicular to the inner photometric axis $\left(\mathrm{PA} \sim 125^{\circ}\right)$. The apex of the biconical structure is located $\sim 2.6^{\prime \prime} \mathrm{NW}$ from the optical nucleus and would be nearly coincident with the origin of the radial motions. We propose that this point is the location of the hidden nucleus of NGC 2992. Notice that it is also sited in a very symmetrical location within the dust lane, as can be inferred from the continuum maps (see Fig. 3d). However, the presence of the dust lane crossing the galaxy could be masking a different location. The hidden nucleus is offcentred with respect to the kinematic center of the disc, but it could be coincident with the kinematic center of the bulge. However, we cannot estimate the location of the latter.

\section{Summary and conclusions}

We have studied the inner $(r<2 \mathrm{kpc})$ circumnuclear region of the interacting Seyfert 2 galaxy NGC 2992 on the basis of new integral field spectroscopic data. Apart from continuum and ionization maps, we have obtained the velocity field for the stellar component as well as those corresponding to the main ionized gas components.

We reach the following conclusions:

1 - Apart from the two main kinematic components for the ionized gas (rotation + outflow) already reported by other authors, an additional kinematic component is required to explain the departure of the ionized gas from the regular behaviour defined from the stars. This component seems to be related to the AGN, and not to the interaction. 
2 - The spatial correlation between an extended arcshaped emission detected in [O III], the figure-of-8-shaped emission in radio, and the kinematic departures from the stellar behaviour, favour the model of an expanding bubble, the ionization being due to shock + precursors models.

3 - The hidden Seyfert nucleus is probably located at the apex of the biconical ionization structure, coincident with the outflow origin and may be with the centre of the bulge. However, the hidden nucleus does not seem to coincide with the kinematic centre of the disc.

Acknowledgements. The authors acknowledge the useful comments of Jose Acosta Pulido. Thanks are also due to Luis Cuesta for making the GRAFICOS code available. We also appreciate the help of Terry Mahoney in editing and correcting the manuscript.

The $4.2 \mathrm{~m}$ William Herschel Telescope is operated by the Isaac Newton Group at the Observatorio de Roque de los Muchachos of the Instituto de Astrofísica de Canarias. The authors thank all the staff at the Observatory for their kind support.

This work has been partially supported by the Spanish Dirección General de Investigación Científica y Técnica (PB93-0658).

\section{References}

Allen, C. W. 1973, Astrophysical Quantities, 3rd Ed. (London: Athlone)

Allen, M. G., Dopita, M. A., Tsvetanov, Z. I., \& Sutherland, R. S. 1999, ApJ, 511, 686

Alonso-Herrero, A., Simpson, C., Ward, M. J., \& Wilson, A. S. 1998, ApJ, 495, 196

Arribas, S., Mediavillas, E., García-Lorenzo, B., del Burgo, C., \& Fuensalida, J. J. 1999, A\&AS, 136, 189

Arribas, S., Carter, D., Cavaller, L., et al. 1998, SPIE, 3355, 821
Arribas, S., Mediavilla, E., \& García-Lorenzo, B. 1996, ApJ, 463, 509

Arribas, S., \& Mediavilla, E. 1993, ApJ, 410, 552

Bingham, R. G., Gellatly, D. W., Jenkins, C. R., \& Worswick, S. P. 1994, SPIE, 2198, 56

Chapman, S. C., Simon, L. M., Alonso-Herrero, A., \& Falcke, H. 2000, MNRAS, 314, 263 (CMAH)

Colbert, E. J. M., Baum, S. A., Gallimore, J. F., et al. 1996, ApJS, 105, 75

Colina, L., Fricke, K. J., Kollatschny, W., \& Perryman, M. A. C. 1987, A\&A, 178, 51 (CFKP87)

del Burgo, C., Mediavilla, E., \& Arribas, S. 2000, ApJ, 540, 741

Dopita, M. A., \& Sutherland, R. S., 1995, ApJ, 455, 468

García-Lorenzo, B., Arribas, S., \& Mediavilla, E. 2000, in Proceedings of Imaging the Universe in Three Dimensions: Astrophysics With Advanced Multi-Wavelength Imaging Devices, ASP Conf. Ser., 195, 325

García-Lorenzo, B., Mediavilla, E., \& Arribas, S. 1999, ApJ, 518,190

Glass, I. S. 1997, MNRAS, 292, L50

Heckman, T. M., Butcher, H. R., Miley, G. K., \& van Breugel, W. J. M. 1981, ApJ, 247, 403

Keel, W. C. 1996, ApJS, 106, 27

Márquez, I., Boisson, C., Durret, F., \& Petitjean, P. 1998, A\&A, 333, 459 (MBDP98)

Nelson, C. H., \& Whittle, M. 1995, ApJS, 99, 67

Osmer, P. S., Smith, M. G., \& Weedman, D. M. 1974, ApJ, 192, 279

Osterbrock, D. E. 1989, Astrophysics of Gaseous Nebulae and Active Galactic Nuclei (Mill Valley CA: University Science Books)

Peletier, R. F., Vazdekis, A., Arribas, S., et al. 1999, MNRAS, 310,863

Ulvestad, J. S., \& Wilson, A. S. 1984, ApJ, 285, 439

Veilleux, S., Shopbell, P. L., \& Miller, S. T. 2001, 121, 198 (VSM01)

Veilleux, S., \& Osterbrock, D. E. 1987, ApJS, 63, 295

Ward, M., Penston, M. V., Blades, J. C., \& Turtle, A. J. 1980, MNRAS, 193, 563 